\title{
Session 1: Cancer
}

Monday 16 September 2002, Moderators: Zdenka L. Jonak and Mark C. Glassy

[09.30-09.50]

PAM-1, a human monoclonal IGM antibody usefull for diagnosis and therapy of precancerous and cancerous epithelial lesions

H. Peter Vollmers, Stephanie Brändlein, Frank

Hensel, Ines Beyer, Matthias Eck, Bernd Schmaußer and Hans Konrad Müller-Hermelink

Pathologisches Institut, Universität Würzburg, Josef-Schneider-Str. 2, 97080 Würzburg, Germany

Many adenocarcinomas of the digestive tract develop from premalignant lesions, induced by bacteria, chemicals or nutritional components. Early detection, differential analysis and treatment of these cancer precursors is very often crucial for survival.

Conventional human hybridoma technology offers the unique possibility to generate new fully human monoclonal antibodies for diagnosis and therapy of diseases and to characterise new tumour related membrane receptors within one experimental approach.

By somatic hybridisation of lymph node B-cells from patients with stomach carcinomas to the heteromyeloma $\mathrm{HAB} 1 / \mathrm{X}$, a fully human germ-line coded monoclonal IgM antibody PAM-1 (Clone 103/51) was isolated. Tested immunohistochemically on paraffin sections, the PAM- 1 antibody reacts with a membrane receptor of nearly all epithelial cancers of all type and origin. Evaluated on non-malignant tissue, the only specific reactivity was found intracellular with proteins in Golgi apparatus.

The receptor was purified from tumor cell membrane extracts and was found to be a $130 \mathrm{kDa}$ integral membrane glycoprotein, homologous to CFR-1 (cysteinerich fibroblast growth factor receptor), which has so far only been detected and described in Golgi of embryonic chicken cells and in $\mathrm{CHO}$ cells. The receptor is homologous to a rat protein, cloned as a Golgi-specific protein, designated MG160, involved in processing and secreting of growth factors. The human homologue, E-selectin binding protein (ESL-1) is a cytokine, expressed on myeloid and some lymphoma cells and modulated by cell adhesion molecules that cause the binding of neutrophils to the endothelium. The PAM1/CFR-1 receptor is, in contrast to the other members of this multi-functional protein family, predominantly expressed on membranes of malignant cells.

Most interestingly, PAM-1 antibody does not only react with epithelial cancers, but also with it's precursors like $H$. pylori induced gastritis, metaplasia and dysplasia of stomach, ulcerative colitis and adenomas of colon and barrett dysplasia of esophagus.

The unique features of PAM- 1 antibody and the unique expression of this new CFR-1 receptor offers the possibility to study specific proliferation processes of malignant and premalignant cells, because the biological function of this CFR family is still unknown. In addition the PAM-1 antibody is also of diagnostic and therapeutical value.

[09.50-10.10]

Multiple strategies for targeting prostate cancer with human antibodies

Nils Lonberg

Medarex, 510 Cottonwood Drive, Milpitas, CA 95035, USA

Human antibodies directed against the cell surface antigens CTLA-4 and PSMA represent potential therapeutic agents for targeting prostate cancer. Medarex is currently developing MDX-101 (high affinity human anti-CTLA-4 IgGk monoclonal antibody) and MDX070 (high affinity human anti-PSMA IgGk monoclonal antibody) for treatment of prostate cancer. These two agents act by very different mechanisms: MDX-010 acts directly on $\mathrm{T}$ cells to stimulate natural immune responses while MDX-070 directly binds to both prostate cancer cells and tumor neovasculature. Data will be presented on the preclinical and early clinical development of these monoclonal antibodies. 
[10.10-10.30]

New human monoclonal IgM antibodies from cancer patients with apoptotic activity

Stephanie Brändlein, Judith Lorenz, Frank Hensel, Matthias Eck, Bernd Schmaußer, Justus Müller, Hans Konrad Müller-Hermelink and H. Peter Vollmers Pathologisches Institut, Universität Würzburg, Josef-Schneider-Str. 2, 97080 Würzburg, Germany

Monoclonal antibodies are accepted to be ideal adjuvant therapeutical reagents for all kinds of diseases. The best source for antibodies against cancerous lesions is the cancer patient itself. Using conventional human hybridoma technique (immortalisation of B-cells from cancer patients by somatic hybridisation to heteromyeloma cells), not only fully human monoclonal antibodies are isolated, but also new targets are identified by the same experimental approach. The resulting antibodies can be used directly for therapeutical purposes without further modulation and manipulation.

We describe here five new established human monoclonal IgM antibodies, CM-1 and CM-2, from a colon cancer patient, PM-1 and PM-2, from a pancreas cancer patient and LM-1, from a patient with a lung carcinoma. The mainly germ-line coded antibodies are specific for malignant tissues and show only restricted reactivity with healthy cells. Tested for functional in vitro activity, all five antibodies inhibit tumour cell proliferation of carcinoma cells by inducing apoptosis. Biochemical studies to determine the corresponding receptors are underway.

Most of the so far described tumor-reacting human antibodies are of IgM type. In addition, these tumorreacting IgM antibodies are germ-line coded or only low mutated. Therefore, these antibodies most likely belong immunologically to the family of natural occuring antibodies and are not the result of a T-cell dependent tumor immunity. Experimental studies by other groups have shown that polyvalent (crosslinking) and low mutated IgM antibodies (less immunogenic) are ideal tools for therapeutical approaches. The natural IgM's isolated from cancer patients are part of the first defense and guarantee that most of the malignant cells are removed at an early stage of development and they are therefore believed to be the most effective weapons against cancer.
[11.00-11.20]

Immunoglobulin repertoire analysis reveals a potential tumor-antigen binding capacity of $B$ lymphocytes infiltrating human breast carcinomas Beatrix Kotlan ${ }^{\mathrm{a}}$, Peter Simsa ${ }^{\mathrm{a}}$, Mehmet K. Tur ${ }^{\mathrm{b}}$, Jean-Luc Teillaud ${ }^{\mathrm{c}}$, Ricarda Finnern ${ }^{\mathrm{b}}$, Gyozo Petranyi $^{\mathrm{a}}$, Rainer Fischer ${ }^{\mathrm{b}}$ and Stefan Barth ${ }^{\mathrm{b}}$ ${ }^{a}$ National Medical Center / National Institute of Haematology and Immunology, Budapest, Hungary ${ }^{\mathrm{b}}$ Fraunhofer IME, Aachen, Germany ${ }^{\mathrm{c}}$ INSERM U255, Universitate P / M Curie, Paris, France

Aim: The objective of the study was to evaluate the tumor specific reactivity of tumor infiltrating B lymphocytes (TIL-B) theoretically and experimentally, as that might be presumed out of some empirical data. The presence of immunocompetent cells in solid tumors may reflect ongoing immune responses against transformed cells that contribute to the spontaneous tumor regression. Contrary to detailed studies about the tumor infiltrating $\mathrm{T}$ cells in terms of $\mathrm{T}$ cell receptor usage and specificity, the tumor infiltrating $\mathrm{B}$ cells, that can be found in a limited number of solid tumors, are poorly investigated.

Methods: About 200 expressed TIL-B immunoglobulin (Ig) heavy and light chain variable regions were cloned and comparatively analysed at nucleotide sequence level from one medullary breast carcinoma, where high B cell and plasma cell infiltration correlates with favourable prognosis. The closest expressed and germline sequences available in EMBL and IMGT databases respectively were defined. A model system was set up in order to reveal the potential capacity of B lymphocytes infiltrating solid tumors to recognize tumor antigens.

Results: The TIL-B immunoglobulin heavy and light chain variable (VH and VL) sequences could be classified into clusters, families and subgroups, based on their high homology levels or identity. We found some clusters composed of overexpressed sequences, that showed about $99 \%$ homology or identity to the DNA sequence of expressed immunoglobulin $\mathrm{VH}$ regions with a proven binding capacity to tumor cells (e.g., disialoganglisosides).

Conclusions: These findings indicate a possible tumor antigen driven selection of singular B cell clones infiltrating solid tumors.

Acknowledgement: This work, - based on earlier findings by a scholarship in France, - was supported by the Hungarian Government (Eötvös Found (XLI/34/2000), OTKA T030380). 


\section{[11.20-11.40]}

Pharmacokinetic analysis of an improved humanized monoclonal antibody designed for radioimmunotherapy (RIT)

Andres Forero, M.B. Khazaeli, Ruby Meredith, Mark Carpenter, Jeff Schlom and Albert LoBuglio

UAB Comprehensive Cancer Center, Birmingham, Al and National Cancer Institute, Bethesda, MD

Since immune response against murine antibodies can limit human use, chimeric and complemetarity determining region (CDR) grafted monoclonal antibodies (MAb) have been generated to solve the immunogenicity hurdle in the clinical application of murine MAbs. However, humanization ( $\mathrm{Hu}$ ) has prolonged plasma circulation in patients, with increased normal tissue retention leading to high background radioactivity and prolonged exposure of the bone marrow to radiolabeled antibody. To overcome these problems a CDR grafted $\mathrm{Hu} \mathrm{MAb}$ with a $\mathrm{CH}_{2}$ domain deletion $\left(\Delta \mathrm{CH}_{2}\right)$ was developed utilizing MAb CC49, which is directed against a pan carcinoma antigen TAG-72 expressed on the majority of colorectal, gastric, breast, ovarian, prostate, pancreatic, and lung carcinomas. The plasma clearance studies in athymic and SCID mice after i.v. or i.p. Administrations demonstrated that $\mathrm{HuCC} 49 \Delta \mathrm{CH}_{2}$ clears more rapidly from the plasma than HuCC49. (Cancer Biotherapy \& Radiopharmaceuticals 12(5):305-316, 1997) We have initiated a phase I RIT trial of ${ }^{131}$ I$\mathrm{HuCC} 49 \Delta \mathrm{CH}_{2}$ in which the first cohort received an imaging/dosimetry dose $(10 \mathrm{mCi}, 20 \mathrm{mg})$. The table below provides mean and standard deviation for pharmacokinetic parameter estimates resulting from a one-compartment bolus model using the NLIN procedure in SAS ${ }^{\circledR}$. The pharmacokinetic analysis of ${ }^{131} \mathrm{I}$ HuCC49 $\Delta \mathrm{CH}_{2}$ and ${ }^{131} \mathrm{I}-\mathrm{mCC} 49$ in humans are compared below:

\begin{tabular}{|c|c|c|c|c|}
\hline & $\mathrm{T} \frac{1}{2}$ (hrs) & MRT (hrs) & $\mathrm{Cl}$ (ml/hr.kg) & AUC \\
\hline $\begin{array}{l}\mathrm{HuCC} 49 \Delta \mathrm{CH}_{2} \\
(n=4)\end{array}$ & $20 \pm 3$ & $29 \pm 4$ & $1.5 \pm 0.1$ & $10.3 \pm 2.2$ \\
\hline $\mathrm{m}-\mathrm{CC} 49(n=13)$ & $50 \pm 11$ & $73 \pm 16$ & $0.9 \pm 0.2$ & - \\
\hline
\end{tabular}

The ${ }^{131} \mathrm{I}-\mathrm{HuCC} 49 \Delta \mathrm{CH}_{2}$ plasma mean $\mathrm{T} 1 / 2$ and MRT were significantly shorter and the mean clearance rate significantly greater than m-CC49 $(p<0.001)$. The whole body and marrow radiation dose estimates were $0.50 \pm 0.06 \mathrm{cGy} / \mathrm{mCi}, 1.00 \pm 0.14 \mathrm{cGy} / \mathrm{mCi}$ with a tumor estimate of $7.4 \pm 1.9 \mathrm{cGy} / \mathrm{mCi}$. The ${ }^{131} \mathrm{I}-$ $\mathrm{HuCC} 49 \Delta \mathrm{CH}_{2}$ biodistribution was similar to m-CC49. None of the patients developed human antibody response to HuCC49 $\Delta \mathrm{CH}_{2}$ after three months of follow- up. HuCC49 $\Delta \mathrm{CH}_{2}$ should have low immunogenicity and improved radioisotope delivery because of its smaller size. The dosimetry estimates indicate that patients will tolerate much higher doses of radiolabeled antibody (higher MTD) with resultant improved tumor radiation doses and probably higher responses. Based on these results, we have recently initiated the dose escalation protocol with ${ }^{131} \mathrm{I}-\mathrm{HuCC} 49 \Delta \mathrm{CH}_{2}$ to evaluate safety, tolerability and MTD.

\section{[11.40-12.00]}

Therapeutic activity of rituximab, a chimaeric anti-CD20 monoclonal antibody, and Fc $\gamma$ RIIIa gene polymorphism

Hervé Watier, Guillaume Cartron, Laurent Dacheux, Gilles Salles, Philippe Solal-Celigny, Pierre Bardos and Philippe Colombat

Laboratoire d'Immunologie, Service d'Oncologie Médicale et Maladies du Sang, Centre Hospitalier Universitaire, Tours, Service d'Hématologie, Centre Hospitalier Lyon Sud, Centre Jean Bernard, Le Mans, France

Background: Rituximab is a chimeric IgG1 antiCD20 monoclonal antibody increasingly used in the treatment of non-Hodgkin's lymphomas. Previous in vitro studies have suggested the role of ADCC and $\mathrm{Fc} \gamma \mathrm{R}+$ effector cells (natural killer cells and macrophages) in the antitumor effects of anti-CD20 antibodies, but the actual mechanism of rituximab action in vivo remains largely unknown. The FCGR3A gene coding for the Fc $\gamma$ RIIIa receptor display a functional dimorphism with either phenylalanine (FCGR3A$158 \mathrm{~F}$ ) or a valine (FCGR3A-158V) at amino-acid 158. The Fc $\gamma$ RIIIa-158V allotype has a higher affinity for human IgG1 and triggers increased ADCC. The aim of this study was thus to determined the influence of this FCGR3A polymorphism on the clinical and molecular responses to rituximab.

Methods: The FCGR3A genotype was determined by a nested PCR followed by allele-specific restriction enzyme digestion (PCR-ASRED) in a population of 49 patients who have received rituximab $\left(375 \mathrm{mg} / \mathrm{m}^{2}\right.$ $\times 4$ ) for an untreated follicular non-Hodgkin lymphoma. The FCGR2A-131H/R genotype was also determined by PCR-ASRED as a control since this gene co-localizes with FCGR3A gene. Clinical and molecular response were evaluated at two months (M2) and at one year (M12). Molecular analysis of the BCL2-JH gene rearrangement was performed by $\mathrm{PCR}$ at $\mathrm{M} 2$ and at M12 and molecular response was defined as a disap- 
pearance of the BCL2-JH gene rearrangement in both peripheral blood and bone marrow. The whole clinical results of this cohort have been published (Colombat et al. Blood 2001;97:101-106).

Results: FCGR3A-158V homozygous patients represented $20 \%$ of the population whereas FCGR3A$158 \mathrm{~F}$ homozygous and heterozygous patients (FCGR3A-158F carriers) were $35 \%$ and $45 \%$, respectively. The objective response rates at M2 and M12 were $100 \%$ and $90 \%(\mathrm{CR}+\mathrm{CRu}=40 \%$ and $70 \%)$ in FCGR3A-158V homozygous patients compared to $67 \%$ (relative risk $=1.5 ; 95 \% \mathrm{CI}, 1.2-1.9 ; P=0.03$ ) and $51 \%$ (relative risk $=1.7 ; 95 \% \mathrm{CI}, 1.2-2.5 ; \mathrm{P}=$ $0.03)$ in FCGR3A-158F carriers $(\mathrm{CR}+\mathrm{CRu}=23 \%$ and $33 \%$ ). A molecular response was observed at M12 in 5/6 of homozygous patient FCGR3A-158V patients compared to $5 / 16$ of FCGR3A-158F carriers (relative risk $=2.8 ; 95 \% \mathrm{CI}, 1.2-6.4 ; P=0.04)$. In multivariate analysis, the homozygous FCGR3A-158V genotype was associated with a greater chance to have a clinical response at $\mathrm{M} 2(P=0.02)$ and at $\mathrm{M} 12(P=0.01)$ as well as molecular response at M12 $(P=0.04)$. On the contrary, FCGR2A-131H/R polymorphism did not influence neither clinical nor molecular responses.

Conclusions: We report here for the first time an easily assessable predictive factor for both clinical and molecular responses to rituximab. This will certainly introduce new pharmacogenetical approaches in the management of NHL patients in order to tailor rituximab treatment to FCGR3A genotype.

[12.00-12.20]

Vn-18 and FibMAb: "Novel antibodies and antigens specific to tumor endothelial cells"

Ton Logtenberg, John de Kruif, Martijn Gebbink and Hetty deBoer

Crucell, Archimedesweg 4, 2301 CA Leiden, The Netherlands

Crucell's Mabstract technology employs scFv phage display libraries to simultaneously detect novel targets and antibodies. In a program focused on tumor endothelial cells and associated extracellar matrix proteins, Crucell identified two novel antibodies, Vn-18 and FibMAb. Both bind specifically to the vasculature of solid tumors without binding healthy tissues, and both have interesting potential as diagnostic and therapeutic antibodies.

FibMAb recognizes a novel tumor-specific epitope of fibronectin. Fibronectin is a molecule which is expressed on the cell surface of several types of differen- tiated cells and is involved in the attachment of cells to the surrounding extracellular matrix.

Vn-18 recognizes a novel activation epitope of vitronectin. Vitronectin, when activated, plays a key role in the attachment, spreading, and migration of endothelial cells and tumor cells. Antibodies binding this protein have been shown to inhibit cellular adhesion in vitro.

\section{[12.20-12.50]}

In Vivo treatment with SB-408075 shows evidence of antibody-toxin delivery to xenografts

Zdenka L. Jonak ${ }^{\mathrm{a}}$, James D. Winkler ${ }^{\mathrm{a}}$, Yi-Jiun Chen ${ }^{\mathrm{a}}$, Francis L. McCabe ${ }^{\text {a }}$, Stephen H. Trulli ${ }^{\mathrm{a}}$, Eian

Caulder $^{\mathrm{a}}$, Lynann M. Inlow-Porter ${ }^{\mathrm{a}}$, Louis Elefante ${ }^{\mathrm{a}}$, Michael Mattern $^{\mathrm{a}}$, Anthony W. Tolcher ${ }^{\mathrm{b}}$, Johann De Bono $^{\mathrm{b}}$ and Randall K. Johnson ${ }^{\mathrm{a}}$

${ }^{\mathrm{a}}$ GlaxoSmithKline Pharmaceuticals, King of Prussia, PA, USA

${ }^{\mathrm{b}}$ Institute for Drug Development, San Antonio, TX, $U S A$

SB-408075, a humanized mAb conjugated to DM1 , a potent small molecule cytotoxic maytansinoid, is currently in Phase I trial. The mAb, huC242, targets a carbohydrate epitope on MUC1 (CanAg), which is expressed on most colorectal (CRC) and pancreatic and some NSCL cancers. In xenografts of human tumors expressing CanAg, SB-408075 produces regressions at doses at or below its MTD. To provide pharmacodynamic endpoints for ongoing clinical trials, we investigated xenografts treated in vivo with SB408075. Immunohistochemistry (IHC) was utilized to detect CanAg (muC242), huC242 ( $\alpha$-human IgG), DM-1 ( $\alpha$-DM-1), and cells in mitosis ( $\alpha$-pMPM- 2$)$. Tumors were excised 1 or 7 days after a single therapeutic dose of $25 \mathrm{mg} / \mathrm{kg}$ of SB-408075. Colo-205 and HT-29 stained strongly for human IgG and showed evidence that DM-1 was delivered to and internalized into these tumor cells. Ab and toxin were still present after 7 days, and there was an increase in mitotic index, although the latter diminished by Day 7 . Colo-205 and HT-29 responded to this treatment with complete regression. Antigen was not saturated by in vivo exposure to humanized $\mathrm{mAb}$ as sections still stained with muC242. In SW-620, there was focal delivery of mAb and toxin to tumor cells at 1 day and antigen binding sites were saturated; an increase in mitotic index was also evident. No DM-1 was detected at Day 7 and there was no tumor regression. In CanAg-neg A-549 neither mAb nor toxin was delivered to tumor cells, 
and SB-408075 did not affect tumor growth. A patient with a sternal metastasis from CRC was biopsied $24 \mathrm{hr}$ after receiving $235 \mathrm{mg} / \mathrm{m}^{2}$ of SB-408075; the tumor stained strongly with $\mathrm{C} 242 \mathrm{mAb}$ and showed delivery and internalization of DM-1. 